\title{
Primary Nasal Tuberculosis
}

\author{
${ }^{1}$ J S Thakur, ${ }^{1}$ Ishan Chauhan, ${ }^{2}$ Shobha Mohindroo, ${ }^{3}$ DR Sharma, ${ }^{4}$ NK Mohindroo \\ ${ }^{1}$ Registrar, Department of Otolaryngology and Head and Neck Surgery, Indira Gandhi Medical College, Shimla, Himachal Pradesh, India \\ ${ }^{2}$ Assistant Professor, Department of Pathology, Indira Gandhi Medical College, Shimla, Himachal Pradesh, India \\ ${ }^{3}$ P rofessor, Department of O tolaryngology and Head and Neck Surgery, Indira Gandhi Medical College, Shimla, Himachal Pradesh, India \\ ${ }^{4}$ P rofessor and Head, Department of Otolaryngology and Head and Neck Surgery, Indira Gandhi Medical College \\ Shimla, Himachal Pradesh, India
}

Correspondence: J S Thakur, Registrar, Department of Otolaryngology and Head and Neck Surgery, Indira Gandhi Medical College, Shimla, Himachal Pradesh, India, e-mail: anujagdeep@yahoo.co.in

\section{ABSTRACT}

Tuberculosis of nose, nasopharynx and paranasal sinus is extremely rare even in countries with a high incidence of pulmonary disease. We present two cases of primary nasal tuberculosis managed in last three years in our department with 2500 to 3000 patient visits per month. One case simulated basal cell carcinoma while other case was an ATT defaulter. Both the cases were managed on RNTCP guidelines. Nasal tuberculosis needs high level of suspicion and is diagnosed with laboratory support.

Keywords: Nasal tuberculosis, Lupus vulgaris, RNTCP, Zeihl-Neelsen.

\section{INTRODUCTION}

Tuberculosis (TB ) of nose, nasopharynx and paranasal sinus is extremely rare even in countries with a high incidence of pulmonary disease. ${ }^{1}$ Tuberculosis of nose usually occur either secondary to pulmonary tuberculosis or to lupus vulgaris of the facial skin. ${ }^{2}$ Tuberculosis in nose was first described in 1761 by Professor Giovanni M orgagni in Italy while conducting autopsy of a young man with pulmonary tuberculosis, who had ulcerations of the nose, soft palate and nasopharynx. ${ }^{3}$ Tuberculosis of nose may be caused by pulmonary disease or less often may represent primary infection. Primary disease may be caused following digital trauma of nose or as a result of inhalation of infected particles.

We present two cases of primary nasal tuberculosis presented in last 3 years.

\section{METHOD}

Retrospective review of cases with nasal tuberculosis was managed in the Department of Otolaryngology and Head and Neck Surgery, IG M edical College, Shimla, HP in the past three years. The department has about 3000 new visits per month and there were only two cases with primary nasal tuberculosis in the past three years.

\section{PATIENTS AND RESULTS}

\section{Case I}

A 64-year-old female from Malan, Kullu, presented with proliferative lesion on the dorsum of nose with nasal obstruction for last one year (Fig. 1). The lesion was painless with occasional episodes of bleeding. She was a smoker and belonged to low socioeconomic status. Patient belonged to cold and high altitude, and hence had long history of sun exposure.

On examination, there was a proliferative growth covered with necrotic tissue, which bled on removing the crusts. Nasal cartilaginous framework was destroyed. A possibility of malignant growth (basal cell carcinoma) was kept and patient underwent punch biopsy. The histopathological examination revealed tuberculosis (Fig. 2). Patient was investigated for other tubercular foci, i.e. pulmonary tuberculosis, but was negative. The patient was hence treated with RNTCP (Revised National

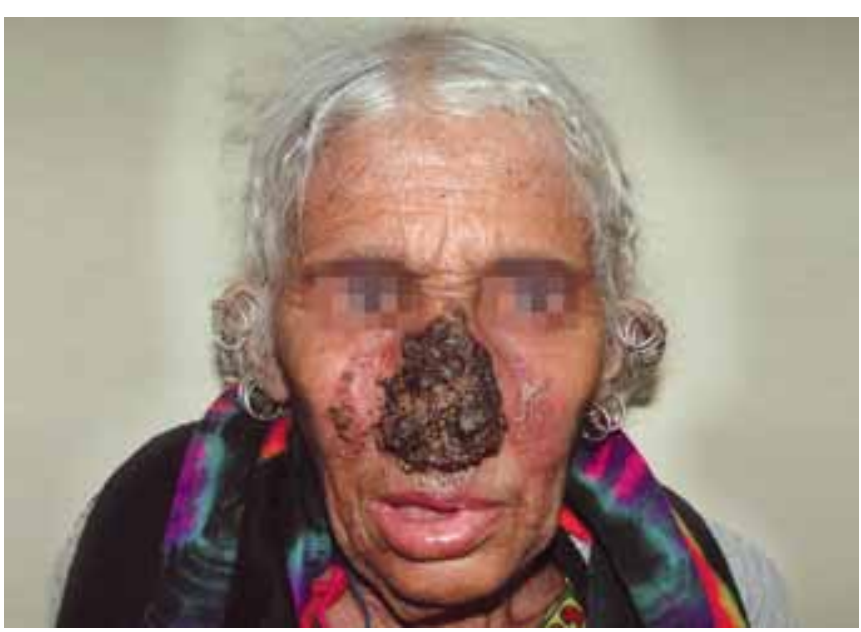

Fig. 1: A 64-year-old female with proliferative growth over nasal dorsum with destruction of cartilaginous framework 


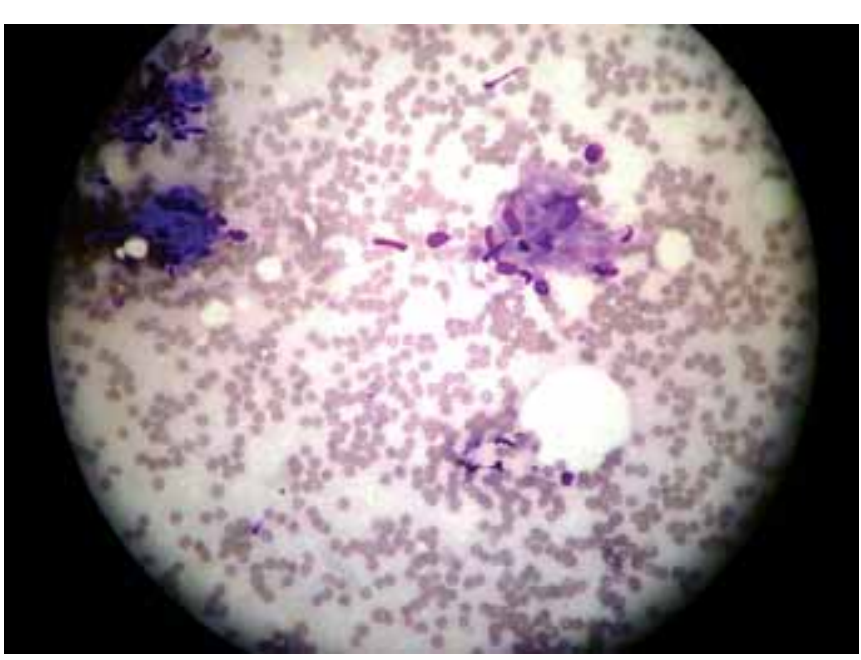

Fig. 2: Microscopy after Ziehl-Nelson staining showing acid fast bacilli

Tuberculosis Control Programme) category III treatment regimen for 6 months. This consisted of an intensive phase of 2 months in which isoniazide $600 \mathrm{mg}$, rifampicin 450 $\mathrm{mg}$ and pyrazinamide $1500 \mathrm{mg}$ were given thrice a week, followed by a continuation phase for the next four months in which, pyrazinamide was withdrawn and other two drugs were continued in same dose. The patient never returned for follow-up.

\section{Case II}

A 12-year-old female child presented with progressive deformity and obstruction of the right nostril for the last one year. She also complained of insidious, painless and progressive swelling in bilateral submandibular region (Fig 3). Patient was earlier diagnosed as a case of nasal tuberculosis 2 years back but did not complete the treatment.

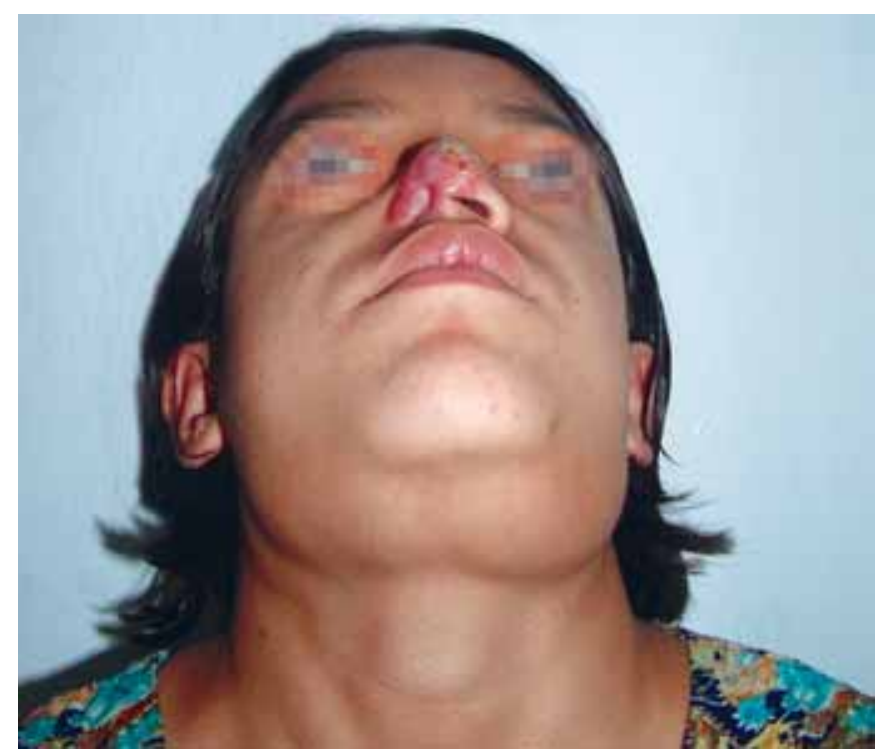

Fig. 3: A 12-year-old girl with ulcerative lesion over right nostril and bilateral submandibular swelling
Local examination revealed deformed nasal alar cartilage. Anterior naris was narrow and filled with crust which bled on removal. There were multiple, matted, firm and painless lymph nodes in bilateral level II. Patient was diagnosed with nasal lymph node tuberculosis.

Routine blood investigations were normal and patient was immunocompetent. Cytology of nasal lesion and lymph nodes confirmed tuberculosis. She was started on R NTCP category II and improved.

\section{DISCUSSION}

The most common symptoms associated with nasal TB are obstruction, damage, epistaxis and crusting. Nasal TB has a variable clinical presentation. Nasal tuberculosis can be classified as spontaneous nasal tuberculosis, which occurs secondarily after pulmonary tuberculosis and as primary nasal tuberculosis, which occurs without a prior pulmonary tuberculosis infection. It has been reported that spontaneous nasal tuberculosis is more common than primary nasal $T B$. ${ }^{4,5}$ This occurs because of the protection afforded by ciliary movements, filtering action of nasal vibrissae and bacteriocidal actions of nasal secretion.,6

$\mathrm{N}$ asal tuberculosis has been reported to occur in the age group of 20 to 84 years with femal es being three times more affected than men. ${ }^{4,5}$ The most commonly involved site is the cartilaginous portion of the nasal septum, followed by the turbinate and nasal floor. ${ }^{4,7}$ Some septal lesions also present with bilateral involvement. ${ }^{8,9}$ Some cases present with septal perforations also. ${ }^{7}$

Onodi described three basic appearances; ulcerative, infiltrative and proliferative. Exophytic, granular lesions are more common than ulcerative lesions, anterior septum is most commonly involved followed by the turbinates and the floor of nose. ${ }^{10-12}$

The differential diagnosis of this disease includes conditions like Wegner's granulomatosis, leprosy, sarcoidosis, subcutaneous phycomycosis, granulomatous syphilis, carcinoma, rhinoscleroma, rhinosporidium seeberi, rhinitis sicca, suppurative sinus disease and foreign bodies, etc. ${ }^{13}$

Establishing the diagnosis is difficult because of the similarity in signs and symptoms of the various other conditions in its differential diagnosis. ${ }^{4,14} \mathrm{~A}$ definitive diagnosis is made by identifying or isolating tuberculous bacilli from tissue removed during biopsy or surgery. Nasal secretions and swab specimens have a very low yield and should not be used to rule out this condition. In histological examination, both caseating and noncaseating granulomas are found and the biopsies of noncaseating granulomas are often confused histologically as W egener's granuloma. In 
tuberculosis, Langerhans giant cells and epithelioid cells are more in number compared to other granulomas. ${ }^{3} \mathrm{~A}$ cidfast bacilli may be demonstrated on Ziehl-N eel sen staining or on auramine-rhodamine staining under fluorescent microscopy. Cultures from biopsy are more sensitive than smears, so a portion of any biopsy specimen should be sent for culture. ${ }^{4}$

Polymerase chain reaction assays, DNA probes and high-performance liquid chromatography are currently recommended only for species identification and to detect primary resistance to drugs, their use is limited because of their high cost, lack of availability and lack of sensitivity. ${ }^{15,16}$

Some patients with nasal tuberculosis subsequently develop pulmonary tuberculosis and/or extrapulmonary tuberculosis in adjacent sites, such as the larynx. ${ }^{4}$ Therefore, the physician should look for these concomitant manifestations during both the initial work-up and subsequent follow-up.

In the pre-chemotherapy era, cauterization of nasopharyngeal or nasal ulceration was done for pain relief along with strict nasal hygiene maintenance using irrigations with alkaline antiseptic solutions and tampons of hydrogen peroxide to soften up the dried crusts. A cids such as lactic, trichloracetic and chromic were used for topical application to the ulcers and galvanocautery and diathermy were done for destruction of the lesions. Other methods, such as use of tuberculin, astringent powders (iodobor and aristol) insufflations in nose, pyrogallic ointment insertion in nose, etc. along with sunlight, $X$-rays or ultraviolet rays, all have been used in past. ${ }^{13}$ In the modern management of the disease, they have little application, as they provide only symptomatic relief.

$\mathrm{N}$ asal tuberculosis should be treated on the general guidelines established for the treatment of extrapulmonary tuberculosis, although variations in the choice of medications may be necessary according to the availability of drugs and the local incidence of resistant strains. According to these guidelines, often three or more drugs are used for a short initial bactericidal regimen followed by a prolonged sterilizing regimen in which at least two drugs are used. ${ }^{15}$

\section{CONCLUSION}

Nasal tuberculosis can simulate malignancy, but histopathological with microbiological investigations confirm the diagnosis. A ntitubercular treatment cures the disease but deformity might need rhinoplasty at later stage.

\section{REFERENCES}

1. L au SK, K wan S, Lee J, Wei WI. Source of tubercle bacilli in cervical lymphadenopathy: A prospectivestudy. J Laryngol Otol 1991;105:558-61.

2. Howard D. Nonhealing granulomas. In: Mackay IS, Bull TR (Eds). Scott Brown's Otolaryngology: R hinology (6th ed). Oxford: Butterworth and Heinemann, 1997;4/20:1-11.

3. W aldman SR, Levine HL, Sebek BA, et al. Nasal tuberculosis: A forgotten entity. Laryngoscope 1981;91:11-16.

4. Goguen LA, Karmody CS. N asal tubercul osis. Otolaryngol Head N eck Surg 1995;113:131-35.

5. Butt A A. N asal tuberculosis in the 20th century. A m J M ed Sci 1997;313:332-35.

6. M CK ibben BG, Sheehy JL. Tuberculosis of the middle ear and nasal passages.A rch Otolaryngol 1956;63:286-89.

7. Chodosh PL, Willis W. Tuberculosis of the upper respiratory tract. Laryngoscope 1970;80:679-96.

8. Sharan R. Primary tuberculosis of the nose. Practitioner 1981;225:1506-07.

9. Chamberlain W. Nasal tuberculosis. A nn L aryngol R hinol Otol 1922;31:423-29.

10. Havens FZ. Primary tuberculosis of the nasal mucous membranes. A rch Otolaryngol 1931;14:181-86.

11. Chamberlain W B. Nasal tuberculosis. A nn L aryngol R hinol Otol 1922;31:423-29.

12. Harper J. Tuberculomata of the nose: Report of two cases. J L aryngol 1919;34:81-82.

13. Dixit R, Dave L. Primary N asal T uberculosis. L ung India 2008; 25(2):102-03.

14. Hughes RG, Drake Lee A. Nasal manifestations of granulomatous disease. Hosp M ed 2001;2:417-21.

15. Raviglione MC, O'B rien RJ. Tuberculosis. In: Braunwald E, Fauci A S, K asper DL, etal (Eds). Harrison's Principles of Internal M edicine (15th ed). N ew Y ork: M cG raw Hill 2001:1024-34.

16. Johnson IJM, Soames (Jr), M arshall HF. Nasal tuberculosis. A n increasing problem? J Laryngol Otol 1995;109:326-27. 